\title{
Le rôle de la reine dans la tolérance entre les ouvrières d'Apis mellifica mellifica
}

\author{
E Saleh-Mghir *, R Darchen \\ Université Paris-VI Pierre et Marie Curie, Station biologique, 24620 Les Eyzies, France
}

(Reçu le 16 mars 1989; accepté le 20 décembre 1989)

\begin{abstract}
Résumé - L'intolérance et le comportement agressif des ouvrières d'Apis mellifica mellifica vis-àvis des organismes étrangers à la ruche sont bien connus. Mais, dans des circonstances particulières, les ouvrières de ruches différentes peuvent s'accepter et cohabiter. Nous avons recherché les facteurs capables d'arrêter le déroulement du comportement agressif des abeilles vis-à-vis des étrangères à la colonie. Nous démontrons ici que la présence de la reine est l'un d'entre eux par son apport capital à l'odeur de la colonie.
\end{abstract}

Apis mellifica / agressivité / reconnaissance de parenté / odeur de la colonie / reine

\section{INTRODUCTION}

Les manifestations agressives des ouvrières d'Apis mellifica vis-à-vis des étrangers à la ruche sont connues de tous. Cette agressivité se manifeste d'ailleurs aussi bien à l'égard des mammifères, des insectes, qu'à l'égard d'abeilles de la même espèce mais d'une autre population. En conséquence, l'apiculteur est obligé d'inventer de nombreux stratagèmes pour tromper les gardiennes des ruches lorsque son travail requiert la réunion de plusieurs populations.

En France, il n'est pas rare d'observer des réunions naturelles de jeunes essaims en quête d'une demeure fixe. Une seule reine se trouve rapidement à l'intérieur de la ruche à la suite de combats mortels. Le nombre des victimes des combats entre ouvrières est alors minime. En Afrique, il est fréquent de découvrir, suspendus à des arbres, des regroupements d'essaims d'une longueur de plusieurs mètres et contenant des dizaines de reines vierges et fécondées (Silberrad, 1976).

Les apiculteurs savent depuis longtemps que, sur une rangée linéaire de ruches, les populations extrêmes s'enrichissent d'abeilles aux dépens de celles du centre. Il est donc clair que, dans des circonstances particulières mais naturelles, les ouvrières de ruches différentes peuvent s'accepter et vivre en paix. Nous avons donc décidé de rechercher en laboratoire quelques-uns des facteurs susceptibles de bloquer le déroulement du comportement agressif des ouvrières d'Apis mellifica mellifica vis-à-vis des étrangères à la société.

\section{MATÉRIEL ET MÉTHODES}

Nous réalisons toujours nos expériences à l'aide de petites populations d'abeilles ouvrières de 75 individus, de tous âges, de la même

\footnotetext{
* Correspondance et tirés à part.
} 
ruche. Les ouvrières utilisées sont donc toujours des "sœurs". Ces ouvrières sont maintenues dans des cagettes de bois de $18 \times 14,5 \times$ $5 \mathrm{~cm}$, vitrées sur les 2 côtés, doublées intérieurement par un morceau de grillage pour permettre aux abeilles de s'accrocher. Un nourrisseur est garni d'un mélange de miel et de sucre glace. Un abreuvoir d'eau pure est à la disposition des insectes.

Les cagettes sont déposées dans une étuve obscure à une température variant entre $29^{\circ} \mathrm{C}$ et $32^{\circ} \mathrm{C}$. Dans ces conditions, la mortalité des abeilles est faible et leur comportement normal durant les 7 jours de l'expérimentation. Nous avons choisi la durée de 7 jours, car l'observation nous a montré que l'activité des abeilles reste stable au cours de cette période. Sur une période plus longue, la vitalité de ces petites populations diminue rapidement malgré tous les soins que l'on peut apporter à leur élevage.

Les abeilles capturées dans les ruches sont marquées par une tache de peinture et séparées sans narcose préalable en 2 cagettes, l'une avec la reine et l'autre orpheline. Nous avons attendu $72 \mathrm{~h}$ entre le moment de la mise en cagettes des abeilles et la première observation, afin de permettre aux insectes de s'adapter à leur nouvel environnement. Lors de l'introduction dans la cagette $X$ de 5 ouvrières provenant de l'autre cagette $Y$ et inversement, nous avons utilisé l'agressivité des abeilles receveuses visà-vis d'une seule abeille choisie parmi les 5 abeilles introduites ensemble comme test de reconnaissance. II est en effet impossible de suivre les réactions comportementales des 5 individus en question et des ouvrières hôtes qui tentent d'établir des contacts avec eux.

Pour quantifier nos résultats, nous avons classé les comportements des receveuses de la manière suivante :

$n^{\circ} 1$ : comportement pacifique entre les ouvrières receveuses et l'ouvrière introduite. Les différentes abeilles se tapotent mutuellement les antennes et effectuent des échanges trophallactiques;

$n^{\circ} 2$ : examen par les abeilles hôtes du corps de l'intruse à l'aide des antennes, s'accompagnant d'ouverture et de fermeture des mandibules;

$n^{\circ} 3$ : les receveuses (parfois une seule) grimpent sur l'abeille introduite;

$n^{\circ} 4$ : les ouvrières receveuses mordent les ailes, les pattes de l'ouvrière introduite;

$n^{\circ} 5$ : combats et mise à mort de l'intruse.
Chaque série d'observations durait $10 \mathrm{~min}$, quotidiennement pendant 7 jours. Enfin, les expériences étaient répétées 10 fois de suite au moyen de 10 couples de cagettes ( $X$ et $Y$ ), peuplées d'abeilles toujours issues de la même ruche (des abeilles sœurs). Cinq reines fécondées, entourées de leurs propres filles, ont servi chacune 2 fois pour réaliser les 10 séries d'expériences.

Pour introduire nos abeilles dans les cagettes hôtes, nous avons employé un tunnel à piston manuel (Saleh-Mghir, 1987).

Nous avons utilisé le test statistique de Wilcoxon-Mann-Whitney sur la somme des rangs (échantillons indépendants).

\section{RÉSULTATS}

\section{$1^{\text {re }}$ série}

Les abeilles sont de tous âges, "sœurs" et orphelines pour les 2 ruchettes $X$ et $Y$ (tableau 1). Dans toute cette série d'expériences, on peut remarquer l'absence totale de comportement agressif entre les abeilles receveuses et les abeilles introduites. La reconnaissance est immédiate car, ensuite, on n'assiste à aucun autre des comportements agressifs codifiés. Donc, les abeilles sœurs, sans contacts avec des odeurs étrangères se reconnaissent pendant plusieurs jours après leur séparation. Le test statistique de Wilcoxon montre que les 2 séries d'expériences (abeilles $X$ transférées en $Y$ et abeilles $Y$ transférées en $X)$ donnent des résultats superposables $(P>15 \%$ pour adopter l'hypothèse nulle). Donc il n'y a pas d'évolution au cours du temps.

\section{0 série}

Les abeilles sont de tous âges et "sœurs". L'une des cagettes $(Y)$ contient une reine fécondée de la même origine que 
Tableau I. Expériences avec 2 populations sœurs orphelines, $X, Y: 2$ cagettes expérimentales. 1, 2, $3,4,5$ : divers types de comportement décrits dans le texte, voir "Matériel et Méthodes", p 70. Les chiffres indiqués dans les colonnes $(1,2,3,4,5)$ sont les moyennes des 10 expériences pour chaque tableau, MOY X en Y, MOY Y en X.

\begin{tabular}{|c|c|c|c|c|c|c|c|c|}
\hline & \multirow{2}{*}{$\begin{array}{c}\text { Nombre } \\
\text { d'abeilles } \\
\text { introduites }\end{array}$} & \multirow{2}{*}{$\begin{array}{l}\text { Transfert } \\
\text { d'une cagette } \\
X \text { à une } \\
\text { cagette Y et } \\
\text { inversement }\end{array}$} & \multicolumn{5}{|c|}{ Comportement } & \multirow{2}{*}{$\begin{array}{l}\text { Nombre d'ouvrières } \\
\text { tuées au cours de } \\
\text { l'expérimentation }\end{array}$} \\
\hline & & & 1 & 2 & 3 & 4 & 5 & \\
\hline \multirow{2}{*}{$1^{\text {er }}$ jour } & 5 & $X$ en $Y$ & 18 & 0 & 0 & 0 & 0 & 0 \\
\hline & 5 & $Y$ en $X$ & 12 & 0 & 0 & 0 & 0 & 0 \\
\hline \multirow{2}{*}{$2^{\theta}$ jour } & 5 & $X$ en $Y$ & 14 & 0 & 0 & 0 & 0 & 0 \\
\hline & 5 & $Y$ en $X$ & 11,5 & 0 & 0 & 0 & 0 & 0 \\
\hline \multirow{2}{*}{$3^{e}$ jour } & 5 & $X$ en $Y$ & 12,2 & 0 & 0 & 0 & 0 & 0 \\
\hline & 5 & $Y$ en $X$ & 14,6 & 0 & 0 & 0 & 0 & 0 \\
\hline \multirow{2}{*}{$4^{\theta}$ jour } & 5 & $X$ en $Y$ & 12,6 & 0 & 0 & 0 & 0 & 0 \\
\hline & 5 & $Y$ en $X$ & 13,5 & 0 & 0 & 0 & 0 & 0 \\
\hline \multirow{2}{*}{$5^{e}$ jour } & 5 & $X$ en $Y$ & 14,9 & 0 & 0 & 0 & 0 & 0 \\
\hline & 5 & $Y$ en $X$ & 13,4 & 0 & 0 & 0 & 0 & 0 \\
\hline \multirow{2}{*}{$6^{\ominus}$ jour } & 5 & $X$ en $Y$ & 14,5 & 0 & 0 & 0 & 0 & 0 \\
\hline & 5 & $Y$ en $X$ & 13 & 0 & 0 & 0 & 0 & 0 \\
\hline \multirow{2}{*}{$7^{e}$ jour } & 5 & $X$ en $Y$ & 16,9 & 0 & 0 & 0 & 0 & 0 \\
\hline & 5 & $Y$ en $X$ & 13,9 & 0 & 0 & 0 & 0 & 0 \\
\hline
\end{tabular}

les ouvrières, l'autre $(X)$ est orpheline (tableau II).

Les résultats sont dissymétriques en ce sens que les réactions des receveuses visà-vis des intruses ne sont pas les mêmes selon que les receveuses ont une reine ou non et ceci bien que les abeilles proviennent d'une même ruche mère.

La reine joue un rôle important. Sa présence dans la cagette $Y$ (c'est-à-dire du côté des receveuses) empêche l'agressivi- té de celles-ci de se manifester trop violemment à l'égard des intruses. En revanche, l'agressivité des abeilles orphelines à l'égard d'une intruse venant de la ruchette à reine est fatale dans 5 cas sur 7 à l'intruse. On note cependant une diminution de l'agressivité au cours du temps. Le test statistique de Wilcoxon comparant les 2 séries d'expériences confirme le phénomène qui, déjà à première vue, semblait évident $(P<0,01 \%$ pour rejeter l'hypothèse nulle). 
Tableau II. Expériences avec 2 populations sœurs. L'une des cagettes $(Y)$ contient une reine fécondée de la même origine que les ouvrières. Dans l'autre $(X)$, les ouvrières sont orphelines. $1,2,3,4,5$ : divers types de comportement décrits dans le texte, voir "Matériel et Méthodes", p 70. Les chiffres indiqués dans les colonnes $(1,2,3,4,5)$ sont les moyennes des 10 expériences pour chaque tableau, MOY X en Y, MOY Y en X.

\begin{tabular}{|c|c|c|c|c|c|c|c|c|}
\hline & \multirow{2}{*}{$\begin{array}{c}\text { Nombre } \\
\text { d'abeilles } \\
\text { introduites }\end{array}$} & \multirow{2}{*}{$\begin{array}{c}\text { Transfert } \\
\text { d'une cagette } \\
X \text { a une } \\
\text { cagette Yet } \\
\text { inversement }\end{array}$} & \multicolumn{5}{|c|}{ Comportement } & \multirow{2}{*}{$\begin{array}{l}\text { Nombre d'ouvrières } \\
\text { tuées au cours de } \\
\text { l'expérimentation }\end{array}$} \\
\hline & & & 1 & 2 & 3 & 4 & 5 & \\
\hline \multirow{2}{*}{$1^{e r}$ jour } & 5 & $X$ en $Y$ & 16,2 & 1,3 & 0 & 0 & 0 & 0 \\
\hline & 5 & $Y$ en $X$ & 4,4 & 15,9 & 12,7 & 14,5 & 0,94 & 4,7 \\
\hline \multirow{2}{*}{$2^{\theta}$ jour } & 5 & $X$ en $Y$ & 15 & 0,7 & 0 & 0 & 0 & 0 \\
\hline & 5 & $Y$ en $X$ & 3,3 & 14,6 & 16,4 & 13,5 & 0,88 & 4,4 \\
\hline \multirow{2}{*}{$3^{e}$ jour } & 5 & $X$ en $Y$ & 15,4 & 1,2 & 0,7 & 0 & 0 & 0 \\
\hline & 5 & $Y$ en $X$ & 4,1 & 11 & 17,6 & 16,9 & 0,82 & 4,1 \\
\hline \multirow{2}{*}{$4^{\theta}$ jour } & 5 & $X$ en $Y$ & 15 & 1,3 & 0,8 & 0,4 & 0 & 0 \\
\hline & 5 & $Y$ en $X$ & 5,3 & 14,6 & 13 & 9,7 & 0,66 & 3,3 \\
\hline \multirow{2}{*}{$5^{e}$ jour } & 5 & $X$ en $Y$ & 19,2 & 1 & 0,7 & 0,1 & 0 & 0 \\
\hline & 5 & $Y$ en $X$ & 5,1 & 11,7 & 10,5 & 12,8 & 0,36 & 1,8 \\
\hline \multirow{2}{*}{$6^{\theta}$ jour } & 5 & $X \in \operatorname{Y}$ & 13,6 & 0,8 & 0,6 & 0 & 0 & 0 \\
\hline & 5 & $Y$ en $X$ & 8,4 & 2,8 & 4,2 & 5,2 & 0 & 0 \\
\hline \multirow{2}{*}{$7^{e}$ jour } & 5 & $X$ en $Y$ & 17,1 & 0,8 & 0,4 & 0,4 & 0 & 0 \\
\hline & 5 & $Y$ en $X$ & 10,9 & 1,6 & 0,7 & 0,6 & 0 & 0 \\
\hline
\end{tabular}

\section{DISCUSSION}

Ces expériences nous font donc découvrir 4 phénomènes importants : 1) la reconnaissance aisée entre ouvrières orphelines et sœurs issues d'une même ruche longtemps après leur séparation (série 1), 2) le rejet d'ouvrières scurs qui viennent de cohabiter seulement quelques heures (72) avec une reine sœur fécondée, 3) l'acceptation totale d'ouvrières orphelines par des ouvrières sœurs de la même ruche mais vivant avec une reine mère fécondée, 4) la diminution de l'agressivité des abeilles orphelines vis-à-vis des intruses non orphelines après quelques jours d'expérimentation.

Chaque phénomène demande une explication : 
L'absence d'agressivité entre ouvrières. Notre surprise a été très grande de la constater, car des auteurs comme Lecomte (1968) ont écrit qu'après 72 h de séparation des ouvrières sœurs ne se reconnaissent plus et s'attaquent violemment. Pourtant, tout l'environnement expérimental est favorable à une reconnaissance permanente entre les 2 petites populations : les cagettes sont semblables et sont abritées dans une même étuve, la nourriture distribuée quotidiennement provient du même stock. Nos observations corroborent le fait : à environnement identique, les critères de reconnaissance demeurent identiques. Les résultats contraires aux nôtres (Lecomte, 1968) avec les mêmes conditions d'élevage proviennent sans doute d'un artefact expérimental lié à l'introduction brutale des abeilles dans les colonies voisines comme semblent le prouver nos travaux antérieurs (Saleh-Mghir, 1987).

Les ouvrières orphelines ne reconnaissent plus leurs sœurs avec reine $72 \mathrm{~h}$ après la séparation. Ceci s'explique par le fait que la reine doit marquer ses congénères très rapidement (moins de $72 \mathrm{~h}$ ) de ses phéromones. Rappelons que chez l'abeille les ouvrières servent de "messagers de l'odeur royale" (Seeley, 1979), ces ouvrières ont de longs contacts avec la reine (supérieurs à $30 \mathrm{~s}$ ) et de nombreux échanges trophallactiques. Elles marchent ensuite rapidement et ont de nombreux contacts antennaires avec les autres ouvrières. Elles sont très actives durant les $30 \mathrm{~min}$ suivant le contact avec la reine (Seeley, 1979). Ce marquage pourrait être la cause des attaques violentes infligées par les abeilles receveuses restées orphelines.

Les ouvrières avec reine reçoivent assez pacifiquement leurs sœurs restées orphelines. Les abeilles imprégnées de l'odeur de leur reine et qui se reconnaissent entre elles devraient rejeter ces intruses qui commencent à moins leur ressembler : leur odeur est différente puisqu'elles ne sont pas marquées par la reine. Pourquoi donc sont-elles acceptées après les explorations sensorielles classiques ? Les abeilles hôtes les reconnaissent-elles encore comme sœurs ? Les étrangères adoptent-elles des attitudes de soumission perçues par leurs hôtes ? Les chiffres de la deuxième série d'expériences nous indiquent en effet que les comportements de reconnaissance (code $n^{\circ} 1$ ) sont beaucoup plus fréquents dans ce type d'expériences que dans l'autre. En revanche, les valeurs des données sont inversées pour le comportement (code $n^{\circ} 2$ ). L'agressivité diminue régulièrement dans le premier cas, elle va en augmentant dans le deuxième. Les chiffres indiquent que, dès le début de la rencontre entre les hôtes et les étrangères, les contacts sociaux jouent un rôle déterminant pour la suite du déroulement de reconnaissance ou d'agressivité des abeilles.

Cette conclusion nous entraîne immédiatement à rechercher si les premiers contacts entre les receveuses et leurs hôtes sont qualitativement et quantitativement les mêmes lorsque les orphelines de la première série d'expériences reçoivent pacifiquement leurs sœurs orphelines ou bien lorsque les ouvrières accompagnées de leur reine de la deuxième série accueillent leurs sœurs orphelines. Cela revient à comparer nos observations comportementales (codifiées $n^{\circ} 1$ ) du tableau I (14 observations de transfert) et du tableau II (7 observations de transfert de $X$ en $Y$ ). Le test de Wilcoxon donne une probabilité $<0,01 \%$ qui permet de rejeter l'hypothèse nulle, c'est-à-dire la similitude des comportements (code $n^{\circ} 1$ ) des 2 séries d'expériences. Ainsi, les abeilles orphelines qui reçoivent leurs sœurs orphelines se comportent différemment des ouvrières vivant avec leur reine et qui assimilent 
leurs sœurs orphelines. D'ailleurs, les comportements (codes $n^{\circ S} 2,3$ et 4 ) de ces dernières sont nettement différents durant les minutes qui suivent les observations du début de l'expérience.

La diminution de l'agressivité au cours des jours d'expérimentation dans le cas où les abeilles, vivant avec une reine, sont attaquées ou même tuées par la population receveuse. Elle peut être expliquée par le fait que :

- le nombre des individus diminue dans les cagettes;

- l'odeur des abeilles introduites et encore vivantes au bout de 4 jours entraîne inévitablement un mélange de populations responsable d'un mélange d'odeurs dans l'atmosphère de la cagette, empêchant par là-même, la détection de l'intruse par les habitants.

Cette étude met en évidence l'importance de la présence de la reine sur la tolérance entre ouvrières d'Apis mellifica.

\section{DISCUSSION ET CONCLUSION GÉNÉ- RALE}

Les sociétés d'insectes sociaux sont souvent caractérisées par des relations hiérarchiques de dominance. Dans les populations d'abeilles (Apis mellifica L), l'individu dominant est la reine, tandis que les ouvrières sont considérées comme formant une caste subordonnée (Wilson, 1975). Mais cette caste subordonnée peut ne pas être homogène. Ainsi Korst et Velthuis (1982) ont montré que des relations hiérarchiques de dominance existaient chez les ouvrières d'Apis mellifica capensis Esch. Ainsi, lorsque la société est orpheline, les ovaires des ouvrières dominantes se développent rapidement. Ces ouvrières produisent alors plus d'œufs et d'acide céto-9 décène 2 oïque (le composé principal de la substance royale). Elles ont donc une valeur adaptative génétique supérieure à celle des subordonnées (Moritz et Hillesheim, 1985).

Wilson (1971), Hölldobler et Michener (1980) ont ainsi émis l'hypothèse que, chez les insectes sociaux, la reine pourrait être à l'origine de l'odeur coloniale, au moins dans les sociétés monogynes. Par ailleurs, d'une façon générale, les sociétés monogynes de fourmis sont plus fermées que les sociétés polygynes (Hölldobler et Wilson, 1977). Wilson (1971) rappelle que les ouvrières de fourmis deviennent tolérantes aux autres individus si elles sont privées de leur reine. II cite l'exemple des sociétés de fourmis Eciton, normalement très hostiles entre elles, mais qui néanmoins fusionnent très rapidement avec celles qui ont perdu leur reine. Dans un travail récent, mené chez Cataglyphis cursor, on a pu observer que les sociétés sans reine acceptaient mieux les individus étrangers (Lenoir et al, 1987).

Les travaux de Benoît (1972) sur Camponotus vagus vont dans ce sens. L'auteur montre que la présence de la reine est impliquée dans les mécanismes de fermeture des sociétés. La fusion de 2 sociétés est beaucoup plus lente lorsque les reines sont présentes, et la fusion complète ne peut avoir lieu qu'à la condition qu'une des reines soit mise à mort. Provost (1979) observe sensiblement la même chose chez les fourmis du genre Leptothorax. Carlin et Hölldobler (1983) ont montré que chez diverses espèces de Camponotus, c'est la reine qui est responsable de la reconnaissance hétérospécifique. On a peu d'informations au niveau homospécifique, mais on peut imaginer que le processus est le même. Toutefois, Mintzer (1982) a montré de façon difficilement réfutable que la reine n'était pas responsable de l'odeur coloniale chez Pseudomyrmex ferruginea. 
Chez l'abeille domestique, Kalmus et Ribbands (1952) ont montré que l'odeur coloniale est influencée par le type de nourriture ingérée par l'individu. Chez cette même espèce, l'existence de facteurs d'origine génétique pour les signaux de reconnaissance a été mise en évidence (Breed, 1981, 1983; Getz et Smith, 1983; Boch et Morse, 1982). Selon Breed (1983) les signaux seraient plutôt d'origine génétique qu'environnementaux. Pratiquement, les signaux endogènes et exogènes pourraient se mélanger en proportions différentes ou bien être utilisés séparément. II émet les hypothèses suivantes : l'odeur environnementale permettrait une reconnaissance globale de la société, ou bien l'odeur environnementale corrélée à celle du génome permettrait dans la société la discrimination entre les membres des diverses lignées paternelles.

Plus récemment, Breed et al (1988) modulent leurs affirmations antérieures en démontrant que l'odeur "génétique" des ouvrières qui se manifeste $12 \mathrm{~h}$ après l'émergence de l'imago peut être facilement masquée par l'odeur du nid.

Deux de nos principaux résultats, le mélange aisé des 2 petites sociétés «sœurs" séparées depuis plusieurs jours et l'acceptation facile d'abeilles orphelines par leurs sours et leur reine après une longue séparation, s'expliquent aisément par leur reconnaissance parentale lorsque toutes les conditions expérimentales restent égales. En revanche, le dernier de nos résultats, à savoir le rejet de "sœurs" vivant avec une reine par des abeilles séparées depuis plusieurs jours, est beaucoup plus difficile à interpréter. En effet, les conditions expérimentales sont les mêmes, l'environnement aussi, les ouvrières sont "sœurs" (il devrait $\mathrm{y}$ avoir une reconnaissance parentale), la reine est leur mère et pourtant les abeilles sont radicalement rejetées. Quel est donc le facteur responsable de cet ac- croc à la règle de la reconnaissance parentale ? Que s'est-il passé entre la séparation des 2 sociétés et leur réunion ? Le temps est trop court pour invoquer la formation d'ouvrières pondeuses dans la société orpheline qui ne recevait même pas de pollen. II nous semble donc que le facteur responsable prend son origine chez la reine seule. Les ouvrières vivant avec elle seraient réimprégnées en permanence par des odeurs émanant d'elle, tandis que les ouvrières orphelines verraient les leurs disparaître.

L'importance de la reine au sein de la société d'Apis mellifica avec le mélange d'odeurs qu'elle véhicule sur le comportement des ouvrières est donc manifeste.

\section{Summary - The queen part in the tole- rance between worker bees, Apis mellif-} era mellifera. The intolerance and the aggressive behaviour of worker bees towards alien individuals of the hive are widely known. But, in particular circumstances, worker bees of different hives can live together without fighting. Thus, it seemed interesting to look for factors which can stop the aggressive behaviour of the honeybee towards alien individuals. Basic to our understanding of these behaviours is the effect of the colony odour. However, colony odour may have various origins, including the hive materials and metabolic products of the workers or the queen. We orientated our research towards investigating the effects of the queen's metabolism.

Experiments were made with populations of 75 workers of various ages taken from the same hive and kept in cages. Seventy-two $h$ elapsed between the time when the workers were put in cages and the first observation. Five workers were introduced from cage $X$ into cage $Y$, and vice versa, and the aggressiveness of the receiver workers towards one worker cho- 
sen amongst the five workers introduced was classified from 1 (no aggressiveness) to 5 (struggle and killing of the intruder). Every set of observations lasted $10 \mathrm{~min}$ and was repeated daily for 7 days.

We demonstrated that the queen's odour played an important part in the colony odour. We now know that : 1) queenless sister workers readily recognize one another, even a long time after their separation (table 1); 2) workers living with a queen easily accept their queenless sisters (table II: lines " $X$ en $Y$ "); 3) queenless workers reject their sisters that have lived with a queen for $72 \mathrm{~h}$ (table II: lines " $Y$ en $X ")$. The two first results may be explained by aspects of kin recognition but not the last one. We hypothesize that queenless workers slowly lose their queen's odour, while workers with queens are continuously marked by the queen pheromone.

\section{Apis mellifera / aggressiveness / kin recognition / colony odour / queen}

\section{Zusammenfassung - Die Bedeutung der Königin für die Verträglickeit zwischen den Arbeiterinnen der Dunklen Biene, Apis mellifera mellifera.} Intoleranz und aggressives Verhalten der Arbeitsbienen gegenüber stockfremden Bienen sind allgemein bekannt. Unter bestimmten Umständen aber können sich die Arbeiterinnen aus verschiedenen Völkern zusammenfinden und ohne Kampf miteinander leben. Es schien uns von Interesse, nach den Faktoren zu suchen, welche die Auslösung eines aggressiven Verhaltens der Stockbienen gegenüber Fremdlingen verhindern. Unser Problem steht ohne Zweifel mit dem Stockgeruch in Zusammenhang; dieser kann aber verschiedenen Ursprungs sein: 1) aus der Umgebung (Wachs, Propolis, Futtervorräte); entstanden aus Beiträgen der
Einzelbienen, die zusammen eine charakteristische Mischung für das Volk ergeben; 3) Abgegeben von der Königin. Mit diesem letzten Punkt befassen sich unsere Untersuchungen.

Die Versuche wurden mit kleinen Gruppen in Käfigen von 75 Bienen aller Altersstufen, aus demselben Volk stammend, durchgeführt. Das Futter war für alle Käfige dasselbe (Honig gemischt mit Zucker, reines Wasser). Zwischen der Bildung dieser Gruppen und der ersten Beobachtung haben wir $72 \mathrm{~h}$ gewartet. Dann wurden 5 Arbeiterinnen aus dem Käfig $X$ dem Käfig $Y$ zugesetzt, und umgekehrt. Dann wurde die Aggressivität der Bienen des Käfigs gegenüber einer ausgewählten Biene aus der Gruppe der fünf zugesetzten nach folgendem Schema klassifiziert: " 1 ", friedliches Verhalten, bis "5", Kämpfe bis zum Tode der zugesetzten Biene. Jede Beobachtungsserie dauerte $10 \mathrm{~min}$, mit täglicher Wiederholung 7 Tage lang.

Unsere Ergebnisse zeigen den wesentlichen Beitrag der Königin am Volksgeruch. Gegenwärtig wissen wir Folgendes: 1) Die Wiedererkennung zwischen weisellosen Geschwistergruppen ist selbst lange Zeit nach der Trennung leicht (Tabelle I); 2) Arbeiterinnen, die mit einer begatteten Königin leben, nehmen ihre weisellosen Schwestern leicht auf (Tabelle II; Linien " $X$ in $Y$ "); im umgekehrten Fall jedoch (weisellosen Arbeiterinnen werden einer weiselrichtigen Gruppe zugesetzt; Tabelle II; Linien " $Y$ in $X$ ") greifen die Bienen ihre Schwestern an, selbst wenn diese nur für Stunden (72 h) mit einer begatteten Königin zusammengelebt haben. Die beiden ersten Ergebnisse lassen sich leicht aus der Erkennung zwischen Verwandten (kin recognition) erklären, das dritte jedoch nicht. Wir stellen uns vor, daß die weisellosen Arbeiterinnen allmählich den Geruch ihrer 
Königin verlieren, während die mit ihrer Königin lebenden ständig durch deren Duft markiert sind.

\section{Apis mellifera / Aggressivität / kin recognition / Volksgeruch / Königin}

\section{RÉFÉRENCES}

Benoît A (1972) Etude expérimentale de la fusion entre groupes chez la fourmi Camponotus vagus Scop., mettant en évidence la fermeture de la société. CR Acad Sci Paris 274, 3564-3567

Boch R, Morse RA (1982) Genetic factor in queen recognition odors of honey bees. Ann Entomol Soc Am 75, 654-656

Breed MD (1981) Individual recognition and learning of queen odors by worker honeybees. Proc Natl Acad Sci USA 78, 2635-2637

Breed MD (1983) Nestmate recognition in honey bees. Anim Behav 31, 86-91

Breed MD, Stiller TM, Moor MJ (1988) The ontogeny of kin discrimination cues in the honey bee. Behav Genet 18, 439-448

Carlin NF, Hölldobler B (1983) Nestmate and kin recognition in interspecific mixed colonies of ants. Science 222, 1027-1029

Getz WM, Smith KB (1983) Genetic kin recognition: honey bees discriminate between full and half sisters. Nature 302, 147-148

Hölldobler B, Wilson EO (1977) The number of queens : an important trait in the ant evolution. Naturwissenschaften 64, 8-15

Hölldobler B, Michener CD (1980) Mechanisms of identification and discrimination in social Hymenoptera. In: Evolution of Social Behaviour ( $\mathrm{H}$ Markl, ed) Verlag Chemie Gmbh, 3558
Kalmus H, Ribbans CR (1952) The origin of the odours by which honey bees distinguish their companions. Proc $R$ Soc London B 140, 5059

Korst PJAM, Velthuis HHW (1982) The nature of trophallaxis in honeybees. Insectes Soc 29 , 209-221

Lecomte J (1968) L'agressivité. In: Traité de Biologie de l'abeille. vol 2, (R Chauvin, ed) Masson, Paris, 374-401

Lenoir A, Querard L, Berton F (1987) Colony founding and role of parthenogenesis in $\mathrm{Ca}$ taglyphis cursor ants (HymenopteraFormicidae). In: Chemistry and Biology of Social Insects. (J Eder, H Rembold, eds) Verlag Peperny, München, 260

Mintzer A (1982) Nestmate recognition and incompatibility between colonies of the Acaciaant Pseudomyrmex ferruginea. Behav Ecol Sociobiol 10, 165-168

Moritz RF, Hillesheim E (1985) Inheritance of dominance in honey bees (Apis mellifera capensis Esch.) Behav Ecol Sociobiol 17, 8789

Provost E (1979) Etude de la fermeture de la société de fourmis chez diverses espèces de Lepthothorax et Camponotus lateralis (Hyménoptères, Formicidae). CR Acad Sci Paris 288, 429-432

Saleh-Mghir E (1987) La reconnaissance interindividuelle chez l'abeille Apis mellifica mellifica. Mémoire de DEA Sciences du comportement. Université de Toulouse

Seeley TD (1979) Queen substance dispersal by workers in honey bee colonies. Behav Ecol Sociobiol 5, 391-415

Silberrad EM (1976) Bee-Keeping in Zambia. Apimondia, Bucarest

Wilson EO (1971) The Insect Societies. Harvard University Press, Cambridge, Massachusetts

Wilson EO (1975) Sociobiology. Harvard University Press, Cambridge, Massachusetts 